\title{
PSMA-targeting Agent-Technetium Tc99m EC0652
}

National Cancer Institute

\section{Source}

National Cancer Institute. PSMA-targeting Agent-Technetium Tc99m EC0652. NCI

Thesaurus. Code C136776.

A radioconjug ate composed of 2-[3-(1, 3-dicarboxy propyl)-ureido] pentanedioic acid (DUPA), a prostate-specific membrane antigen (PSMA)-targeting ligand, linked to the radioisotope technetium $\mathrm{Tc} 99 \mathrm{~m}$ ( $\mathrm{T}$ c99m), that can potentially be used as a radioimaging agent for PSMA-overexpressing tumor cells. Upon administration, the PSMA-targeting moiety of EC0652 targets and binds to PSMA-expressing tumors. Upon uptake and single-photon emission computed tomog raphy (SPECT) imaging, PSMA-expressing tumors can be visualized and identified. In turn, PSMA-overexpression can be used to evaluate both the efficacy of and response to certain PSMA-targeting cytotoxic agents. PSMA, a tumor-associated antigen (TAA), is overexpressed by most prostate cancers. 